CORRECTION

https://doi.org/10.1038/s41586-019-0906-z

\title{
Author Correction: Direct observation of incommensurate magnetism in Hubbard chains
}

Guillaume Salomon, Joannis Koepsell, Jayadev Vijayan,

Timon A. Hilker, Jacopo Nespolo, Lode Pollet, Immanuel Bloch \& Christian Gross

Correction to: Nature https://doi.org/10.1038/s41586-018-0778-7, published online 12 December 2018.

In this Letter, author Christian Gross should have been associated with affiliation '1' (Max-Planck-Institut für Quantenoptik, Garching, Germany) instead of affiliation '2' (Fakultät für Physik, LudwigMaximilians-Universität, Munich, Germany); this has been corrected online. 